\title{
LONGITUDINAL AND TRANSVERSE FEEDBACK SYSTEMS FOR PLS STORAGE RING*
}

\author{
H. S. Kang, W. H. Hwang, H. J. Park, D. T. Kim, J.Y. Huang, and S. H. Nam \\ Pohang Accelerator Laboratory, Pohang, 790-784, Kyungbuk, Korea
}

\begin{abstract}
The Pohang Light Source (PLS) storage ring uses two bunch-by-bunch feedback systems - a longitudinal feedback system (LFS) and a transverse feedback system (TFS) - to cure the coupled bunch instabilities due to higher order modes of RF cavities and resistive wall impedance. The LFS employs the digital electronics developed for ALS and PEPII and uses a cavity-type kicker, and it was successfully commissioned in 1999 with the aid of SLAC. The TFS uses analog two-tap coax notch filters for closed orbit offset signal rejection. The TFS is now in upgrade to use one kicker per transverse plane to improve efficiency over the previous system with a kicker combining both planes in a single structure. This paper will describe the achieved results of curing the coupled bunch instabilities by using the LFS and TFS together with current status.
\end{abstract}

\section{INTRODUCTION}

In a third generation light source the strong coupled bunch instability is a big concern, which limits beam current and degrades photon beam quality by amplifying beam oscillation or by blowing up beam emittance.

The design beam currents of the PLS are $400 \mathrm{~mA}$ and $250 \mathrm{~mA}$ at $2 \mathrm{GeV}$ and $2.5 \mathrm{GeV}$, respectively. The achieved maximum stored beam current was $450 \mathrm{~mA}$ at $2 \mathrm{GeV}$, $200 \mathrm{~mA}$ at $2.5 \mathrm{GeV}$. A big longitudinal instability begins to appear at the current higher than $230 \mathrm{~mA}$ at $2.0 \mathrm{GeV}$, above that a peanut-shaped beam is borne, which is apparently useless for beam line users. The limitation of beam current at $2.5 \mathrm{GeV}$ is due to the $\mathrm{RF}$ power limitation of the current RF system.

To minimize the beam coupling impedance in RF cavities for higher current operation, the HOM tuning method is exploited intensively, which includes the fine adjustment of cavity temperature and an insertion of HOM tuning plunger into RF cavity. RF Cavity HOM tuning and chromaticity can reduce the instabilities to some extent, but not enough because there are still residual impedances in a ring not to be removed so that an active bunch-by-bunch feedback system is required to perfectly remove the instabilities. In order to cure the coupled bunch instabilities in transverse and longitudinal directions, two bunch-by-bunch feedback systems have been prepared - a longitudinal feedback system and a transverse feedback system. Longitudinal coupled bunch instability becomes more severe as the stored current increases and transverse instability is getting worse as the complexity of vacuum chambers increases in a ring. Small gap insertion device chambers have a gap distance of 5 to $12 \mathrm{~mm}$, which remarkably increases the transverse resistive wall impedance. Another important thing is that the higher chromaticity, the dynamic aperture becomes much smaller.

In this paper we will describe the test results of the bunch-by-bunch feedback systems and the upgrade plan together with current status.

\section{LONGITUIDNAL FEEDBACK SYSTEM}

To suppress the longitudinal instabilities, the longitudinal feedback system, originally developed for the PEP-II, DAФNE and ALS machines and shares common architectures, was procured from SLAC in 1999 [1]. Table 1 lists the machine parameters of the LFS.

Table 1: Machine parameters of the LFS

\begin{tabular}{|l||c|c|}
\hline \multirow{2}{*}{ Energy, GeV } & 2.0 & 2.5 \\
\hline Bunches & \multicolumn{2}{|c|}{468} \\
\hline Sampling Frequency, MHz & \multicolumn{2}{|c|}{500.063} \\
\hline Revolution Frequency, MHz & \multicolumn{2}{|c|}{1.0685} \\
\hline Synchrotron Frequency, kHz & 11.4 & 10.0 \\
\hline Down-sampling factor & 15 & 18 \\
\hline Tap Number & 12 & 6 \\
\hline Sampling Rate, kHz & 71 & 59 \\
\hline Numbers of DSPs & \multicolumn{2}{|c|}{ Cavity } \\
\hline Kicker type & 1 & 1 \\
\hline Number of kickers & \multicolumn{2}{|c|}{$9 / 4$ RF } \\
\hline Kicker frequency & $250 \mathrm{~W}$ & $500 \mathrm{~W}$ \\
\hline Amp Power & \multicolumn{2}{|c|}{} \\
\hline
\end{tabular}

To damp the longitudinal bunch oscillation, the longitudinal feedback system measures the energy of particle bunches and computes a correction signal from the energy errors, and corrects the energy of the particles. The LFS uses a digital signal processing formalism to compute correction signals. Signals from four button-type pickups are combined and fed to stripline comb generator. The generator produces a four-cycle burst at the sixth harmonic of the ring RF frequency $(3 \mathrm{GHz})$. The resultant signal is phase detected, then digitized at the bunchcrossing rate. A correction signal for each bunch is computed by a digital processing module and applied to the beam through a fast D/A, an output modulator, a power amplifier and a kicker structure.

*Supported by the Ministry of Science and Technology, Korea 
Table 2 shows the setting parameters for the LFS to damp the instabilities, which are measured with the beam current of $135 \mathrm{~mA}$ at $2 \mathrm{GeV}$. The front-end that measures the energy of particle bunches has a limit of synchronous phase motion about 29 degree, which depends on beam current. The back-end delay which times the kick to the exact bunch has a very small stable range, which means a very precise back-end timing is absolutely necessary. On the other hand, the filter and the down sampler that compute a correction signal have a large operation range.

Table 2: Setting parameters for the LFS to damp the instabilities.

\begin{tabular}{|l|l|c|c|}
\hline Module & $\begin{array}{c}\text { Setting } \\
\text { parameter }\end{array}$ & $\begin{array}{c}\text { Operation range } \\
\text { for damping }\end{array}$ & Span \\
\hline \hline \multirow{2}{*}{ Filter } & Phase & $0 \sim 75$ degree & 75 \\
\cline { 2 - 4 } & Shift gain & $3 \sim 4$ & \\
\hline $\begin{array}{l}\text { Down } \\
\text { Sampler }\end{array}$ & $\begin{array}{l}\text { Synchrotron } \\
\text { frequency }\end{array}$ & $10.5 \sim 12.3 \mathrm{kHz}$ & $1.8 \mathrm{kHz}$ \\
\hline $\begin{array}{l}\text { Front- } \\
\text { end }\end{array}$ & Phase shift & $-11 \sim 18$ & 29 \\
\hline \multirow{2}{*}{ Delay } & Back-end & $1597 \sim 1712 \mathrm{ps}$ & $115 \mathrm{ps}$ \\
\cline { 2 - 4 } & Front-end & $5273 \sim 6248 \mathrm{ps}$ & $975 \mathrm{ps}$ \\
\hline
\end{tabular}

The most important parameter is damping rate by feedback. The radiation damping times $\left(\tau_{r}\right)$ in longitudinal direction are $8.3 \mathrm{~ms}$ and $4.2 \mathrm{~ms}$ at $2 \mathrm{GeV}$ and $2.5 \mathrm{GeV}$, respectively. Figure 1 shows the longitudinal feedback damping time $\left(\tau_{F}\right)$ as a function of phase deviation [2]. For 1-degree phase deviation, about $2 \mathrm{~ms}$ damping time by feedback can be achieved with a $250 \mathrm{~W}$ amplifier. To damp the instability the total damping rate should be higher than the instability growth rate $\left(\tau_{i}\right)$ : $1 / \tau_{i}<1 / \tau_{r}+1 / \tau_{F}$. In case of $2.5 \mathrm{GeV}$ the feedback system with a $250 \mathrm{~W}$ amp does not provide a sufficient damping rate compared to the radiation damping. In order to increase the damping rate at $2.5 \mathrm{GeV}$, it is necessary to install another kicker cavity or double the amp power.

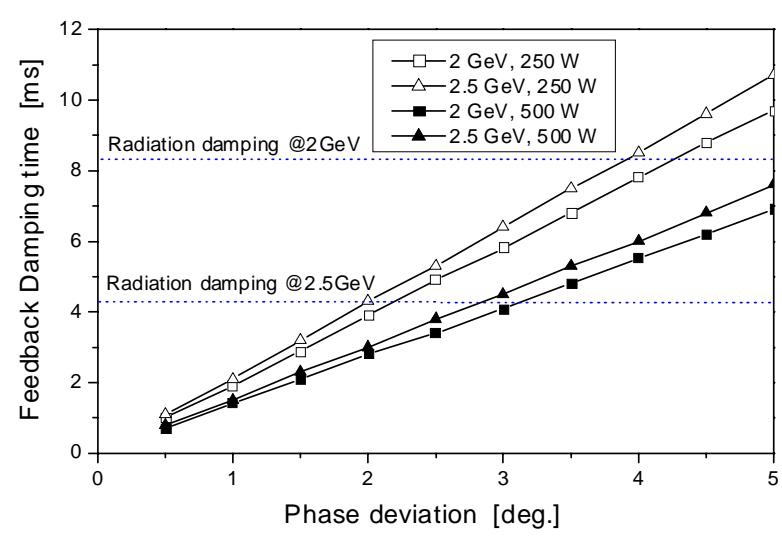

Figure 1: Longitudinal feedback damping time as a function of phase deviation.
The LFS was successfully commissioned at the end of 1999 by the help of SLAC, and a very stable electron beam could be stored up to $240-\mathrm{mA}$ at $2 \mathrm{GeV}$. All longitudinal coupled bunch instabilities disappeared after the LFS turns ON and the phase oscillation amplitude of all bunches decreases to noise level.

The problem we frequently encounter in operation of the LFS is the lack of repeatability and reliability. The bases the LFS functions well are: to measure the accurate phase oscillation amplitude of all bunches and to calculate the appropriate correction value, and the correction kick to the exact bunch. The main causes that deteriorate the basis turned out to be the external fiducial and the RF cavity gap voltage variation, especially during beam injection.

The external fiducial from the timing system to the LFS timing module makes the LFS recognize the first bunch in a bunch train. Recently the timing system can provide the external fiducial signal to the LFS timing module, which enables the LFS to be exactly timed to the right bunch position. The large variation of RF cavity gap voltage occurs during the beam injection. The synchrotron frequency varies with an amount of about $100 \mathrm{~Hz}$ as the RF gap voltage changes during the beam injection. This causes the longitudinal position of the synchronous particle to move, which disturbs the accurate measurement of the phase oscillation amplitude. The variation of cavity gap voltage is due to the low speed of cavity voltage feedback loop of the low-level RF feedback system. The low-level RF feedback system is now in upgrade to incorporate the amplitude and phase feedback loops for klystron and RF cavity, each. The upgrade will be finished by October of 2001. The upgrade system is expected to guarantee the constant gap voltage control.

\section{TRANSVERSE FEEDBACK SYSTEM}

To suppress the transverse instabilities, a hybrid transverse feedback system has been developed. The system uses two pickups for the detection of the betatron oscillation and a single stripline-type kicker for damping in both horizontal and vertical directions. But the transverse feedback system also has the problem of lack of repeatability and reliability. It requires a very finetuning of signal delay whenever the TFS is in use.

The feedback system was tested in both horizontal and vertical direction, separately. After the beam tests the TFS was decided to change the scheme: separates the direction of kicks and uses two kicker chambers of the same design. The new system uses one kicker per transverse plane to improve efficiency over the previous system combining both planes in a single structure. For this scheme, another kicker chamber was installed at the beginning of 2001. The upgrade of the TFS is now in progress, will be finished by the beginning of 2002 .

Figure 2 depicts the block diagram of the new transverse feedback system. The major components are betaron pahse adjuster from two pickup signals, delayline notch filters to reject revolution harmonic signals, and backend power amplifiers. Beam moment, $I \delta x$, is 
detected at $3 f_{r f}$ from two BPM pickups. The momentum signals are demodulated to baseband where kicker efficiency is at maximum with heterodyne receivers. The TFS uses analog two-tap coax notch filters for closed orbit offset signal rejection to avoid amplifier saturation by orbit harmonics. The TFS must feedback on $d x / d t$, transverse momentum, for real damping with the baseband correction signal. Thus the feedback system must include a $90^{\circ}$ phase shift between detection and kick. Corrections are applied approximately one turn after detection, which requires broadband one-turn delays. The kicker is driven by two broad-band $(10 \mathrm{kHz} \sim 250 \mathrm{MHz})$, $75 \mathrm{~W}$ class-A power amplifiers [3].

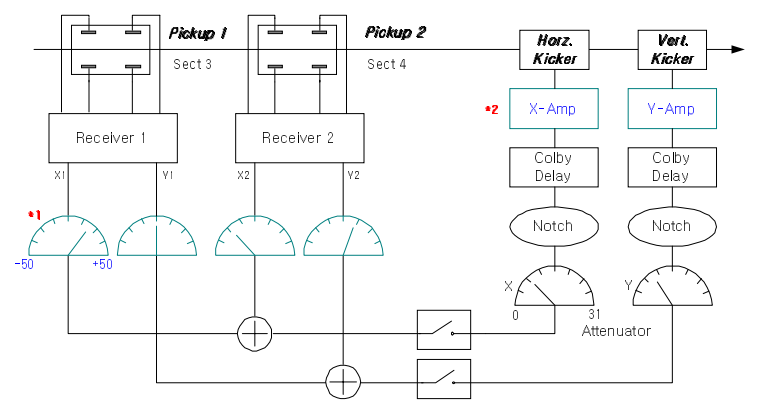

Figure 2: Block diagram of the transverse feedback system.

Figure 3 shows the potential distribution of the kicker structure calculated by the POISSON code for vertical direction and the calculated transverse feedback damping rate as a function of vertical displacement. For 1-mm vertical displacement, the damping rate is about $3000 \mathrm{sec}$ ${ }^{1}$ with two $75 \mathrm{~W}$ amps. The transverse shunt impedance of the kicker is calculated assuming circular shape. Measurement of the transverse shunt impedance of the kicker structure is necessary to know more accurate damping rate.

The beam tests for the transverse feedback system with one kicker per plane were successfully done using one kicker structure, which shows a good repeatability in function. The beam test result has shown a clear damping of beam oscillation as shown in Figure 4, with $30 \mathrm{~dB}$ damping of the beam oscillation in the whole $250 \mathrm{MHz}$ bandwidth. The stripline kicker, $15 \mathrm{~cm}$ long, used for tune measurement is excited by an RF signal generator to induce a transverse oscillation in horizontal or vertical directions. We achieved the damping of $25-40 \mathrm{~dB}$ for vertical direction at the stored current of $145 \mathrm{~mA}$ at $2 \mathrm{GeV}$. Horizontal damping is $10-25 \mathrm{~dB}$, smaller than vertical direction, which is the expected result. When we use the instability generated by beam itself, damping by the TFS is very effective: $30-40 \mathrm{~dB}$ for vertical direction, 50 - 60 $\mathrm{dB}$ for horizontal direction. For this test we use a full bunched beam of $200 \mathrm{~mA}$ to induce ion instability. In this case the growth rate of transverse instability in vertical direction is higher than that of horizontal direction.

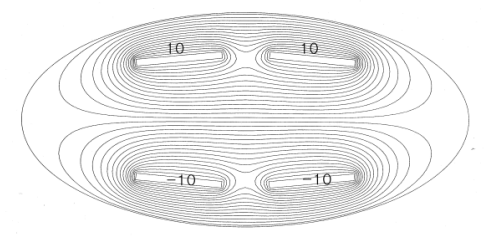

(a)

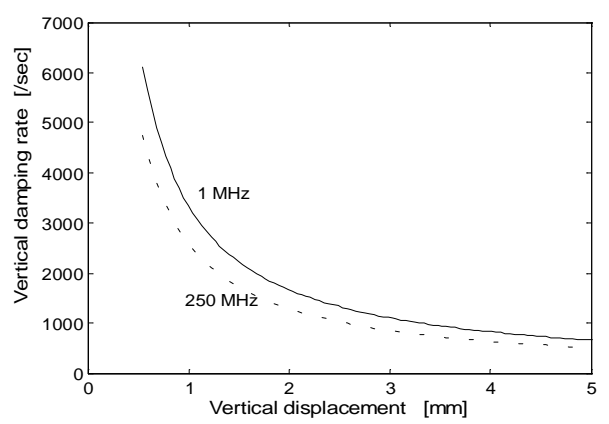

(b)

Figure 3: Potential distribution calculated by POISSON code for vertical direction (a) and the calculated vertical feedback damping rate as a function of vertical displacement.

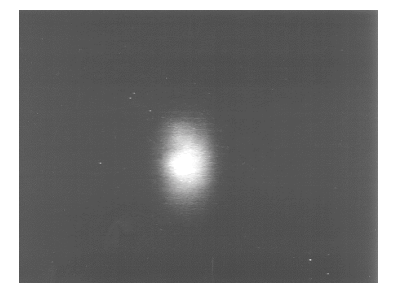

(a)

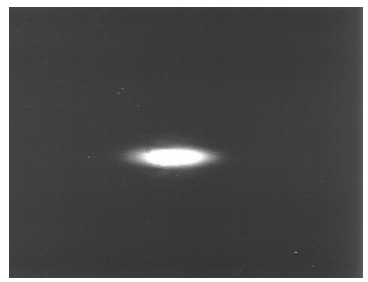

(b)
Figure 4: Visible light images measured at the diagnostic beam line when the TFS in vertical direction is OFF (a) and $\mathrm{ON}(\mathrm{b})$.

\section{SUMMARY}

The performance verification study was carried out for the present bunch-by-bunch feedback systems - LFS and TFS. Based on the test, the performance upgrade plan is now in progress to ensure the repeatability and reliability: further performance test of each module of the LFS, to use one kicker per transverse plane to improve efficiency for the TFS, and a new low-level RF feedback system to ensure the longitudinal motion stability. After the completion of this upgrade, the bunch-by-bunch feedback systems can be used to perfectly damp the instabilities with good reliability.

\section{REFERENCES}

[1] J. Fox, et al., "Programmable DSP-based multi-bunch feedback - operational experience from six installations," BIW, 2000.

[2] S. Khan and T. Knuth, "Longitudinal and Transverse Feedback Systems for BESSY-II".

[3] D. A. Goldberg and G. R. Lambertson, "Dynamic Devices a Primer on Pickups and Kickers", LBL-31664, November 1991. 